\title{
Abdominal wall abscess, an unusual complication of Seton: A case report.
}

\author{
Debasis Naik ${ }^{1}$, Gopalakrishnan $\mathrm{G}^{1}$ \\ ${ }^{I}$ (Post-Graduate, Department of General Surgery, V.M.M.C. and Safdarjung Hospital, New Delhi.)
}

\begin{abstract}
An anal fistula is a communicating tract between the inner anus or rectum and the external skin surrounding the anus. It causes chronic discharge of pus that typically has an offensive odor. The treatment of anal fistula has challenged physicians and healers for millennia. References to fistulous disease and use of both fistulotomy and seton can be found in the writing of Hippocrates, dating from 400 BC. Although the primary objective of operative procedure is to heal the fistula, equally important is the morbidity of the procedure. Seton have been used to manage anal fistula from hundreds of year; however, in the literature, Seton were commonly used only for high or complex anal fistula in order to avoid fecal incontinence or recurrence. The usual complications arising due to a seton procedure are discomfort, bleeding, local abscess formation, mucous or liquid stool discharge, recurrence and incontinence. We present a case of abdominal wall abscess arising as a complication of Seton procedure.
\end{abstract}

Keywords : Seton, abdominal wall abscess, septicemia, high anal fistula.

\section{Introduction}

Fistula-in-ano (anal fistula) is one of the commonly encountered surgical problem with a prevalence of 1.2 - 2.8 per 10,000[1]. Operation for anal fistula is among the oldest surgical procedures, the use of seton to treat an anal fistula being described by Hippocrates[2]. Seton have been used to manage anal fistula from hundreds of year; however, in the literature, Seton were commonly used only for high or complex anal fistula in order to avoid fecal incontinence and recurrence[3]. We report a case of abdominal wall abscess arising as a complication of Seton procedure.

\section{Case Report}

A 56 year old man was admitted with complaints of fever, pain abdomen, distension of abdomen and non passage of flatus and feces for the last 2 days. This was associated with pus discharge per rectum for the last 1 day. He had history of Seton placement 2 months back for high anal fistula. Patient had no other comorbid illness. Patient was dyspneic and Chest examination revealed bilateral course crepitations. On abdominal examination, it was tense, distended and bowel sounds were absent, there was no guarding or rigidity. Seton was found in-situ with active pus discharge per rectum. On Digital rectal examination, it was tender and Empty. His TLC was $42,000 / \mathrm{mm}^{3}$ of which $90 \%$ were polymorphs. X-ray abdomen revealed no air-fluid level or gas under diaphragm and USG abdomen revealed normal study.

Seton was removed and patient was put on higher generation i.v antibiotics. CECT Abdomen was done and Blood Culture was sent. On the 2nd day, patient went into shock and he developed erythema and induration of antero-lateral abdominal wall. CECT report revealed a foci with collection noted surrounding rectum, pelvic floor muscle, extending to anterior pre-peritoneal space and bilateral abdominal wall muscles laterally. A foci was also noted to extend in right side retroperitoneum overlying the right psoas muscle. A collection was also noted along the inner surfaces of bilateral internal oblique muscles with maximum thickness of $1.4 \mathrm{~cm}$ in left side and $1.7 \mathrm{~cm}$ in right side(Fig 1,2,3). Immediate surgical intervention was attempted by giving release incisions in bilateral flanks. Intraoperatively, there was release of $200 \mathrm{ml}$ of foul smelling seropurulent fluid with gush of air. But as the general condition of the patient didn't permit a definitive surgical procedure couldn't be attempted. Post-operatively, the patient went into septicemic shock and expired. Blood culture report received after 72 hours revealed Gram negative bacilli and anaerobes.

\section{Discussion}

Fistula in ano is a chronic abnormal communication usually lined by some degree of granulation tissue which runs outward from anorectal lumen "internal opening" to the external opening on the skin of the perineum or the buttock[4].

According to park's classification, the rate of intersphincteric fistulae reported in literature is $70 \%$. Besides, $24 \%$ of fistulae are transphincteric, 5\% are suprasphincteric and $1 \%$ extrasphincteric [5,6]. 
Patient may present with persistent discharge from the internal and/or external openings which may be purulent or bloody and causes pruritus and discomfort in the surrounding skin[7]. Pain which is increasing until temporary relief occurs when the pus discharges[4]. The majority of anal fistulae are of crypto-glandular origin, following anorectal abscess in $7-40 \%$ of cases. Besides, anal fistulae are sometimes associated to other conditions, mainly inflammatory bowel disease, particularly Crohn's disease[8].

On digital rectal examination, the internal opening may be felt as a point of induration or it may reveal a posterior or lateral induration indicating fistula deep in post anal space or a horse shoe fistula[9]. Anoscopy aids in identifying the internal opening in the anal canal. Sigmoidoscopy or colonoscopy helps to exclude proximal internal opening, inflammatory bowel disease or neoplasia, if the diagnosis is suspected based on the history of recurrent fistulae, history suggestive of inflammatory bowel disease or examination revealed multiple fistulae[4,10,11].

The investigation that can be used for the diagnosis of fistula in ano include MRI which is the gold standard for fistula imaging, the great advantage is its ability to demonstrate the fistula track and presence of any secondary extensions[4,10,11]. Endoluminal ultrasound; especially with hydrogen peroxide can also be used to delineate the fistula. It is helpful to determine whether the fistula is straight forward or not $[12,13]$.

Surgical procedures for high transphincteric fistulas include advancement flap closure, with a different success rate according to the etiology of fistula and a recurrence rate ranging between 0 and $63 \%$. Cutting setons have been used in an attempt to slowly divide the sphincters while allowing scarring to occur and limit disruption of the muscular ring, with recurrence rate from $22 \%$ to $39 \%$. During the last ten years, fibrin glue injection has become a popular alternative to the cutting seton and mucosal advancement flap repair of complex fistulas; however, the published success rates widely between $14 \%$ and $60 \%[14]$.

Seton is any string-like material which when tied through the fistula tract causes an inflammatory reaction which stimulates fibrosis that fixes and prevents retraction of sphincter continuity when it is divided. In this way, it maintains sphincter continuity during continuity process. Different types of Setons are used for this purpose like silastic tube, silk, linen, braided silk, rubber band, braided polyester, vascular loop, polypropylene, nylon, cable tie, and so forth[15].

The usual complications arising due to a seton procedure as mentioned in various literatures are discomfort, bleeding, local abscess formation, mucous or liquid stool discharge, recurrence and incontinence where as there is no mention of Abdominal wall abscess as its complication in literature. Abdominal wall abscess as a complication of Seton procedure is a possibility, which can be diagnosed from the clinical presentation and timely investigations like CECT Abdomen, MRI etc.

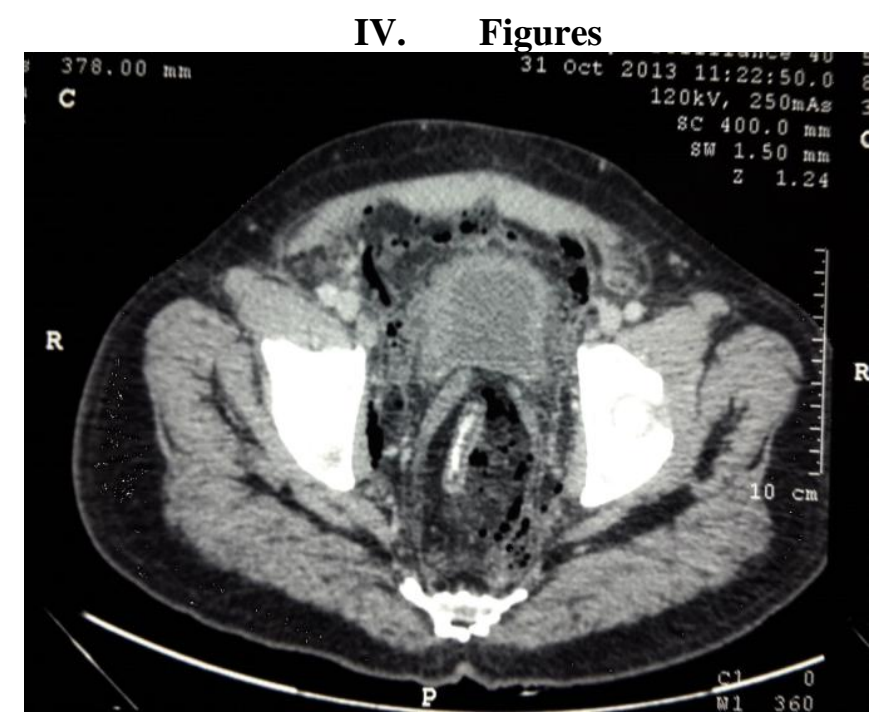

Fig 1 : CECT Abdomen showing collection and air foci around rectum and pelvic floor muscles. 


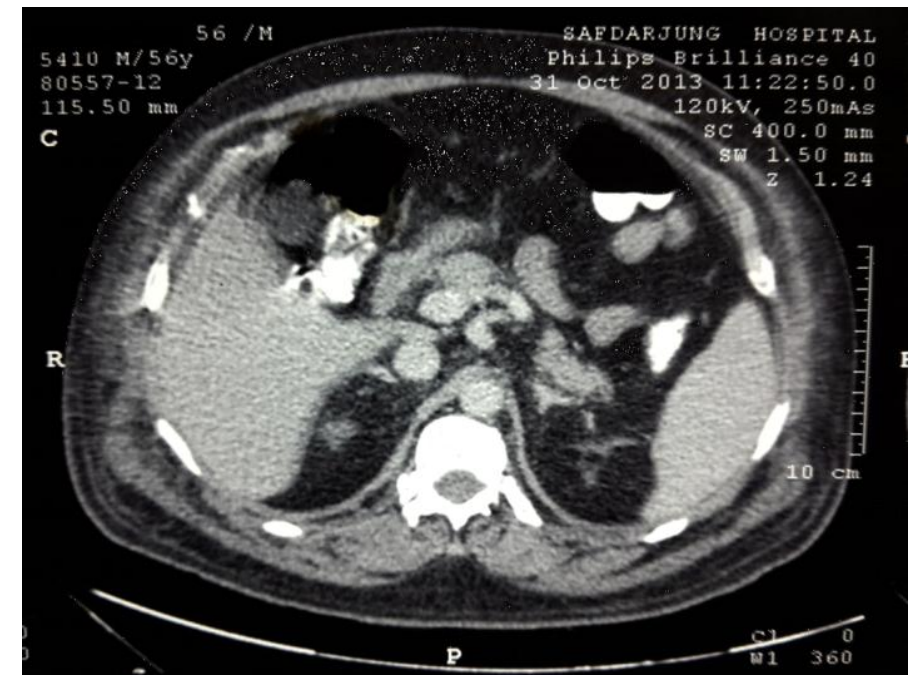

Fig 2 : CECT Abdomen showing collection in Bilateral abdominal wall muscles.

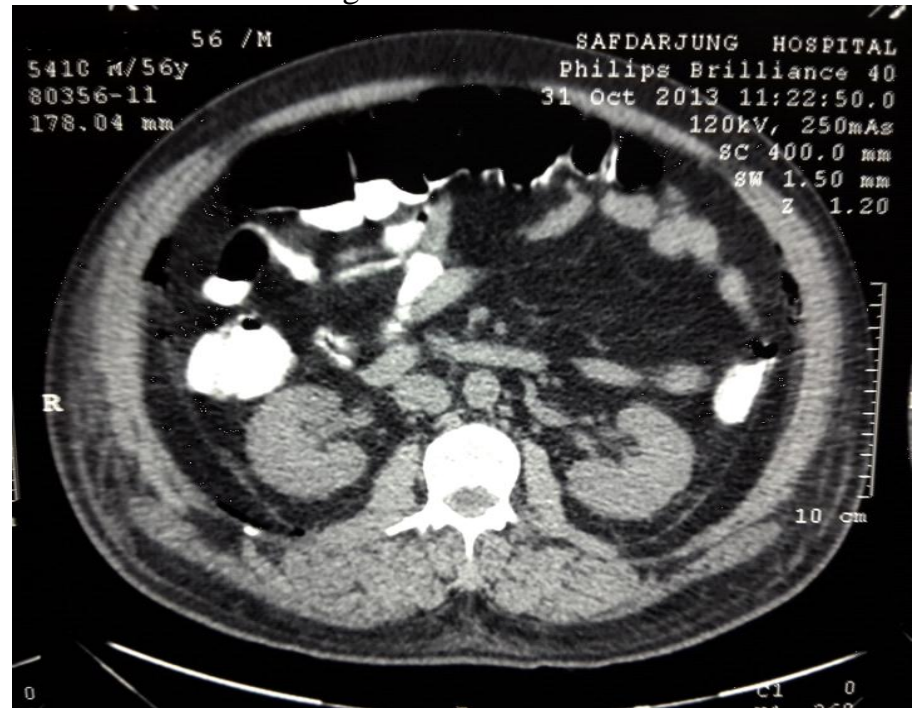

Fig 3 : CECT Abdomen showing collection and air foci in the preperitoneal space.

\section{Conclusion}

In conclusion, any patient presenting with acute abdomen and features of sepsis, with history of seton placement, Abdominal wall abscess as a complication of Seton procedure should be considered as a possibility. Aggressive management is needed to avoid mortality.

\section{Reference}

[1]. Zanotti C, Martinez-Puente C, Pascual I, Pascual M, Herreros D, Garcia-Olmo D. An assessment of the incidence of fistula-inano in four countries of the European Union. International Journal of Colorectal Disease. 2007; 22(12): 1459 - 1462.

[2]. Adams F. On fistulae. In: Adams F, ed. The Genuine work of Hippocrates. Baltimore: Williams and Wilkins, 1939: 337 - 42.

[3]. Pearl R K, Andrews J R, Orsay C P, et al. Role of the Seton in the management of anorectal fistulas. Disease of Colon and Rectum. 1993; 36(6): 573 - 579.

[4]. Bailey and Love's Short Practice of Surgery. Norman. S williams, Christopher J.K. Bulstrode, P.Ronan O Connell. 25th Ed. The anus and the anal canal. 2008; 69: 1262 - 64

[5]. Abcarian H, Dodi G, Gironi J, et al: Symposium-Fistula-in-ano. Int J Colorect Dis. 1987; 2: 51 - 72.

[6]. Christensan A, Nilas L, Christiansen J: Treatment of Transphincteric and fistulas by the seton technique. Dis Colon Rectum. 1986; $29: 454$ - 5.

[7]. Vasilevsky C A. Fistula in ano and abscess Im; Beck D E, Wexner S D, Eds. Fundamentals of anorectal Surgery. 1992: 131 44.(s).

[8]. Sabiston Textbook of Surgery: The Biologic Basis of Modern Surgical Practice. M. Townsend, R. Daniel Beauchamp, B. Mark Evers and Kenneth L. Mattox. 19th Ed. Anus.2013. pp: 1392.

[9]. Kuypres J H. Diagnosis and treatment of fistula in ano. Neth J. surg. 1982; 34: 147 - 52

[10]. Gordon N.Buchanan, Steve Hallingan, Clive I. Bartram, Andrew B. Williams et al. Clinical Examination, Endosonography, and MR Imaging in Preoperative Assessment of Fistula in Ano: Comparison with Outcome-based Reference Standard1. Radiology. 2004; 233: 674 - 81

[11]. Lunniss P J, Barker P G, Sultan A H, et al. magnetic resonance imaging of fistula in ano. Dis Colon Rectum 1994; $37: 708$ - 18. 
[12]. Lindsey, I. Humphreys, M.M., George, B. D. and Mortensen, N. J. M. C., The role of anal utrasound in the management of anal fistulas. Colorectal Disease, 2002; 4: 118 - 22.

[13]. Ratto C, Gentil E, Merico M, Spinazzola C et al. How can the assessment of fistula-in-ano be improved? Dis Colon Rectum 2000; 43: $1375-82$.

[14]. Sileri et al. Surgery for fistula-in-ano in a specialist colorectal unit: a critical appraisal. BMC Gastroenterology 2011; 11: 120.

[15]. Ritchie R D, Sackier J M, Hodde J P. Incontinence rates after cutting seton treatment for anal fistula. Colorectal disease. 2009;11(6): $564-571$. 